\title{
Editorial
}

\section{New strategies in regenerative medicine and surgery and their application to orthopedic surgery field: The bio-active composite therapies (BACTs)}

\author{
Michele Zocchi ${ }^{1} *$ \\ ${ }^{1}$ Plastic and Reconstructive Surgery Unit - University of Padua, Padua, Italy
}

\section{A R T I C L E I N F O}

Article history:

Received 25-10-2021

Accepted 11-12-2021

Available online 12-01-2022

\section{Keywords:}

Bio-Active Composite Therapies (BACTs)

Stromal Vascular Fraction (SVF)

\begin{abstract}
A B S T R A C T
Regenerative Medicine and Surgery is a rapidly expanding branch of translational research in tissue engineering, cellular and molecular biology.

To date, the methods to improve cell intake, survival and isolation need to comply with a complex and still unclear regulatory frame, becoming everyday more restrictive and often limiting effectiveness and outcome of the therapeutic choices. Thus, the author developed a novel regenerative strategy, based on the synergic action of several bio-active components, called the Bio-Active Composite Therapies (BACTs) to improve grafted cells intake and survival in total compliance with the legal and ethical limits of the current regulatory frame. The rationale at the origin of this new technology is based on the evidence that cells need supportive substrate to survive in vitro and this observation, applying the concept of translational medicine, is true also in vivo. Many different sources have been used in the past for MSCs, molecules and growth factors (GF) isolation and extraction, but the Adipose Tissue and its Stromal Vascular Fraction (SVF) definitely remains the most valuable, abundant, safe and reliable. Bio-Active Composite Mixtures (BACMs) are tailor-made injectable "cocktails" containing several bio-active components to support cells survival and induce a strong regenerative response in vivo by stimulating the recipient site to act as an in-situ real Bioreactor. In this article, the author analyze the main causes of cell's death and the strategies for preventing it, and outline all the technical steps for preparing the main components of BACMs and the different mixing modalities to obtain the most efficient regenerative action on different clinical and pathological conditions in several surgical specialties. Orthopedic Surgery is definitely the one that most can benefit of these new therapeutic strategies. The final part of this work is anticipating the logical and sequential evolution toward other fundamental technical steps for further supporting and enhancing the most efficient regenerative activity.
\end{abstract}

This is an Open Access (OA) journal, and articles are distributed under the terms of the Creative Commons Attribution-NonCommercial-ShareAlike 4.0 License, which allows others to remix, tweak, and build upon the work non-commercially, as long as appropriate credit is given and the new creations are licensed under the identical terms.

For reprints contact: reprint@ipinnovative.com

\section{Introduction}

Regenerative Medicine is today considered the newest and most promising global health pillar based on the clinical application of cell therapy by promoting and stimulating body's own repair mechanisms. ${ }^{1}$ Mesenchymal Stem Cells (MSCs), first described by A. Caplan in 1989, have shown a unique potential in repairing damaged tissues and organs

\footnotetext{
* Corresponding author.

E-mail address: info@michelezocchi.com (M. Zocchi).
}

and active regeneration whenever compromised by the most different etiology and reasons, ranging from acute causes and trauma to chronic and degenerative conditions. Cell therapy exhibits unique advantages, such as its ability of restoring tissue function, together with high viability and low morbidity. ${ }^{2-4}$

Medicinal signaling cells (MSCs), previously known as mesenchymal stem cells, are multipotent stromal cells originating from the three mesenchymal layers (exo-dermic, meso-dermic and endo-dermic) able to differentiate into 
many cellular lines such as osteoblasts, chondrocytes, adipocytes, endothelial, muscular and nervous cells. ${ }^{5}$ MSCs are strictly in contact with the basement membrane and with endothelial cells of the microvascular net. During a regenerative process, MSCs become functional and establish a local regenerative microenvironment called Niche, for ensuring metabolic and chemo-tactic exchanges. Specific markers such as NG-2b and CD-146 are expressed both on the surface of isolated MSCs and on Pericytes, a cell population within the microvascular net. ${ }^{6}$

Among different sources of MSCs, Adipose Tissue (AT) remains one of the most promising, valuable and reliable sources of regenerative elements as Adipose-derived Stem Cells (ADSCs). ${ }^{7,8}$

Active tissue healing and Extra Cellular Matrix (ECM) deposition are even promoted by local synergic support of cells, molecules and GFs. In fact the secretome derived from MSCs may contribute to the regeneration of the tissue microenvironment in damaged or injured areas. The MSCs have been shown to secrete bioactive factors that affect immune systems' cells and functions such as inhibition of apoptosis, enhancement of cellular migration, promotion of angiogenesis and increase of the rate of proliferation of stem or progenitor cells present in the tissue in a process called "trophic activity". ${ }^{2}$

Nonetheless, from the pathophysiological point of view, unfortunately many different elements can deeply interfere with transplanted cells survival and local homeostasis and, consequentially, induce Transplanted Cell Death (TCD) within the recipient site: host Inflammatory response, host immune response, shear and mechanic stress, local activity of reactive oxygen species (ROS), hypoxia and low nutrient supply are the most relevant noxia leading to Programmed Cells Deaths (PCD) consequent to anoikis and apoptosis. ${ }^{9}$ Whereas mechanical stimulation and crushing can be at the origin of shear stress, the inflammatory host and immune response are associated with local and systemic cytokines activation; low nutrient supply and hypoxia are key in several metabolic stress processes while PCD for anoikis, due to ECM separation from cellular components, and apoptosis seems to be sustained by abnormal activity of Death-Associated Protein 3 (DAP3). All these elements, working in a deleterious synergism, can consistently jeopardize transplanted cells integration to the host recipient site by interfering with niches homeostasis.

For these reasons since many years now, the author dedicated consistent efforts to analyze and develop new strategies aimed to improve Transplanted Cell Survival (TCS). Tissue engineering, gene transfer and pre-conditioning procedures, ROS protection, Complement Inactivation, cytokines modulation and pro-biotic redirecting showed very promising potential in favoring ADSCs intake, survival and differentiations. ${ }^{10}$
Pro-survival factors are mainly supported and mediated by extracellular membranous vesicles, better known as Exosomes, containing mRNAs and signal molecules, able to mediate intercellular communication by transporting proteins or nucleic acids into target cells, thus altering the behaviors of recipient cells. The combination of all these pro-survival factors sustains, through a positive synergic interaction, niches homeostasis and allows the transplanted therapy survival inside of the host recipient site.

Though, recognizing all the above mentioned factors able to improve cell survival and proliferation made the author determined to reproduce in-vivo in the host's recipient site the same in-vitro cell plate conditions for cells expansion and cultivation to improve grafted cells homeostasis, intake and survival, developing a safe and reliable approach still respecting the existing regulatory frame's limits.

The main concept behind this new line of research is that for obtaining the most efficient Regenerative activity, tuned for every clinical need and for every anatomic area, it is mandatory to use the synergic action of several bio-active components. Instead of injecting a "Single-component Graft" alone (i.e. ADMCs), a multi-component Bio-Active Mixture called BACMs with enhanced regenerative activity can be used to support transplanted cells, kick-starting different regenerative processes and inducing the recipient site to act as an in vivo real Bio-Reactor. ${ }^{11,12}$

\subsection{Composition of the bio-active composite mixtures}

BACMs are mainly composed of two basic bio-active components:

1. Cellular Components (CCs) isolated and extracted from freshly Adipose-derived Stromal Vascular Fraction (SVF), either Tissutal SVF with its ECM fraction or pure and concentrated Cellular SVF with the sole cellular components very rich in ASCs precursors;

2. Blood Components (BCs), such as Platelet Rich Fibrin (PRF) very rich in specific and aspecific GFs.

These two components alone represent, depending on the clinical needs, between 70 to $90 \%$ of their total volume. However other important non bio-active components should be added in minor proportions for completing the BACMs regenerative potential:

1. Type 1 Bio Catalyzers, such as Aminoacids (AA), Vitamins, Reduced Glutathione (GSH);

2. Type 2 Bio-Catalyzers, such as specific Morpho Proteins;

3. Carriers such as HA, Polysaccharides and ECM.

These additional components could be crucial for redirecting, supporting and enhancing the outcome of the two main bio-active components and should be added in 
different proportions and concentration to the BACTs and grafted to the host recipient site. The proportion between SVF, BCs and the other components depends on the clinical situation, on the therapeutic needs and on the recipient site's anatomy and volume. Specific protocols have been established for adapting the bio-active preparation. Table 1.

Table 1: The components of the Bio-active composite Mixtures: Adipose-derived Stromal Vascular Fraction, Blood Components, Bio Catalyzers and Carriers.

\begin{tabular}{|c|c|c|}
\hline \multicolumn{3}{|c|}{ Basic components of the bio-active composite mixtures } \\
\hline $\begin{array}{l}\text { Adipose Tissue } \\
\text { Derivates }\end{array}$ & SVF & MSCs and ECM \\
\hline $\begin{array}{l}\text { Blood } \\
\text { Components }\end{array}$ & PRGF, PL, PRF & Growth Factors \\
\hline $\begin{array}{l}\text { Bio Catalyzers } \\
\text { Type } 1\end{array}$ & $\begin{array}{l}\text { AA, Vitamins, } \\
\text { GSH }\end{array}$ & $\begin{array}{l}\text { Cell Intake and } \\
\text { Growth }\end{array}$ \\
\hline $\begin{array}{l}\text { Bio Catalyzers } \\
\text { Type } 2\end{array}$ & Morpho Proteins & $\begin{array}{l}\text { Linear } \\
\text { Differentiation }\end{array}$ \\
\hline Carriers & HA, PCA, ECM & $\begin{array}{l}\text { Facilitate } \\
\text { Implantation }\end{array}$ \\
\hline
\end{tabular}

\subsection{Cellular Components: stromal vascular fraction and $A D S C s$}

The most common tissue sources of MSCs beside Adipose Tissue are bone marrow, blood, lungs, teeth's dental pulp and different fetal components such as placenta, amniotic fluid and membrane and the umbilical cord. The previous research on MSCs was mainly focused on Bone Marrow Mesenchymal Stem Cells (BMSCs). ${ }^{2}$ However, the discomfort and frequent pain associated with the invasive procedure for harvesting bone marrow and the very limited number of MSCs that can be extracted from this source always represented an important technical limit and it has progressively lost popularity with the sole exception of the Orthopedic Surgery field where it can still find some supporters. Conversely, the adipose tissue can be easily isolated in very large amount from multiple body areas with minimally invasive procedures and is certainly today the most safe, abundant and accessible source of MSCs with significant proliferative and multi-lineage differentiation potential towards several cellular lines (such as adipogenic, osteogenic and chondrogenic). Those cells are contained in a heterogeneous cellular mix, called Stromal Vascular Fraction (SVF) composed of ECM and different key cells types including erythrocytes, lymphocytes, $\mathrm{T}$ regulatory cells, fibroblasts, monocytes, M2 macrophages, pericytes, preadipocytes, endothelial cells and ADSCs precursors. It is possible to identify two different fractions of SVF: the Tissue SVF (TSVF) containing also the ECM component (collagen, laminine, elastine and other proteins) and the Cellular SVF (CSVF) mostly composed by the nucleated cellular pool. This heterogeneous cell population must undergo to a multiparameter flow cytometric assay to identify cells and relative sequences.

The number of MSCs precursors contained in the ATderived CSVF is significantly larger than in the bonemarrow (up to 1000 times more): from $10 \mathrm{cc}$. of decanted AT, with the existing technologies it possible to insulate nearly 1cc. of SVF, containing up to 18.000 .000 of nucleated cells. ${ }^{11}$ However it is important to underline the fact that only 5 to $7 \%$ of this cells pool, in the best scenario, is represented by MSCs progenitors. ${ }^{13,14}$ The author would like to underline the fundamental role of plastic surgeons as the historic experts in the most advanced and safe techniques for harvesting and manipulating adipose tissue for preparing, in close cooperation with other specialists, the most efficient regenerative grafts. As a matter of fact it is author's opinion that, in the aim of obtaining the best possible results meanwhile ensuring the highest safety to the patients, this type of procedures should be carried on by a multidisciplinary team where clinical indications and grafting procedures into the different recipient sites are performed by each appropriate and trained specialists only.

Before to enter into the technical details related to composition and preparation of BACMs and in order to avoid any confusion, the author would like to stress an important preliminary concept: the term Mesenchimal Stem Cells (MSCs) and Adipose Derived Stem Cells (ADSCs) too often are erroneously used by non qualified professionals mainly for commercial purposes. Those acronyms should be used to identify only and solely those cultured cells after expansion, isolation and cultivation. All the other cellular or tissue fractions obtained either with enzymatic digestion or with mechanical disruption of AT, without undergoing any of the above mentioned laboratory processes, should be instead identified as "freshly insulated SVF" containing a small fraction of MSCs precursors and Pericytes.

\subsection{Blood components}

The second key Component of the BACTs is represented by Blood Components (BC) containing patient's own blood platelets, which act as natural source of specific and aspecific GFs (e.g. PDGF, IGF, VEFG, PDAF, TGFBeta and many others). The release of GFs is triggered by the activation of platelets and can be kick-started by different substances such as thrombin, calcium chloride and collagen. Blood derived GFs support chemotaxis, proliferation, differentiation and angiogenesis in a highly controlled way by releasing different molecules (e.g. fibronectin, vitronectin and sphingosine 1-phosphate). However, different compositions of BC type offer different features, GFs concentrations and therapeutic actions, ${ }^{15,16}$

Red and Yellow Platelet-Rich Plasma (PRP), High Concentration Amber PRP (HCAPRP), Plasma Rich in Growth Factors (PRGF) Platelet Lysate (PL) or Platelet Rich Fibrin (PRF) are most common used blood components. ${ }^{17,18}$ 
Among them, red PRP has been widely used in the past, given its easy extraction method and affordable cost. However, its richness in red and white cells is at the origin of significant cytokine activation and enhancement of local inflammation at the point that can be even contraindicated in some specific pathologies, such as OsteoArthritis (OA) Type 1 where the increased inflammatory response can worsening the clinical situation.

PRGF and PL can be considered as enhanced types of $\mathrm{BC}$, with a higher pool of GFs, if compared to red or plain amber PRP, containing a higher quantity of GFs and inducing a better anti-inflammatory response. PRGF and PL can be added to freshly isolated SVF whenever a high concentration of regenerative elements inside of a small volume is required. However, even the clinical use of PRGF and $\mathrm{PL}$ is certainly more valuable and efficient then red or plain amber PRP, these BC are still less common due their very high, and often unjustified cost of the isolation device and of the related kits (Magellan/Regennex).

It is authors' modest opinion that today the most appealing and innovative BC is certainly PRF, a slow release autogenous matrix fibrin gel product that can be considered a three-dimensional (3-D) structure that favors the delivery and support of cell sheets. Unlike platelet-rich plasma, PRF can be obtained from blood using low-speed centrifugation without adding any type of chemical or anticoagulants. The procedure used by the authors does not require any special automated device and expensive kits but just a simple system using a centrifuge a very inexpensive kits (Next PRP and PRX by TLab - Turkey). In less than 10 minutes and with minimal cost, from $20 \mathrm{cc}$ of blood it is possible to obtain from 4 to $6 \mathrm{cc}$ of PRF only alternating repetitive spins at different $G$ (from 3 to 5 ) and without using jellifying agents. Cytokines, GFs and cells are mixed together in a homogeneous multi-factorial pool where the different factors can be slowly released from PRF over time (from 3 to 18 days). PRF is very rich in EGF, FGF and specific pro-inflammatory cytokines such as IL-1b, IL-6 and TNF-alfa and trough a strong secretomic action it's able to affect the genetic and cellular activity, playing a significant role in the inflammatory response of the grafted biomaterial to the recipient site. ${ }^{19}$

\subsection{Bio catalyzers}

A complete cell culture Medium is composed of a basal medium containing low-molecular-weight substances such as inorganic ions, amino acids, vitamins and other additional components (e.g. glucose, pyruvate). Due to the fact that medium composition is often inadequate for the in-vitro homeostasis of different cell lines, high-molecular weight supplements (e.g. proteins) have to be added to fulfill cell requirements. However, even if confident of the intrinsic safety of this strategy, because of the still unclear oncogenic role of cancer stem cells, in some oncological patients we are limiting the use of high-molecular components.

There are two different types of Bio Catalyzers, which can be added to the BACTs [Table 2].

Table 2: Amino acids, Vitamins, GSH and high-molecular weight morphogenic proteins are added to the Regenerative Mixture to support cell's intake and survival in the recipient site

\begin{tabular}{|c|c|c|}
\hline \multicolumn{3}{|c|}{ Bio Catalyzers Type Action } \\
\hline Type 1 & $\begin{array}{l}\text { Aminoacids, } \\
\text { Proteins, GSH }\end{array}$ & $\begin{array}{l}\text { Ability of mimicking in vivo } \\
\text { the action of DMEM Medium }\end{array}$ \\
\hline Type 2 & $\begin{array}{l}\text { Morphogenic } \\
\text { proteins }\end{array}$ & $\begin{array}{l}\text { Stimulation and redirection } \\
\text { of cells towards specific } \\
\text { cellular lines }\end{array}$ \\
\hline
\end{tabular}

\subsection{Bio catayzers type 1}

By adding Aminoacids (AA) and Vitamins (VITs) to the cellular and blood components it is possible to reproduce in vivo the same biological action of a Dulbecco Modified Eagle Medium (DMEM) in vitro for expansion and cultivation. Reduced Glutathione (GSH) antagonizes ROS deleterious activity.

\subsection{Aminoacids}

AA improves and support protein synthesis, presenting a key role in mammalian cells cultivation and homeostasis. ${ }^{20}$ With the aim of mimicking the supportive role of a DMEM it is useful to enrich BACTs with a pool of 12 essential L aminoacids such as arginine, cysteine, leucine, isoleucine, lysine, methionine, phenylalanine, threonine, tryptophan, histidine, tyrosine and valine. These additional components working in synergism with the other components support cells' intake and growth to the host recipient site. ${ }^{20}$

\subsection{Vitamins}

VITs act as cofactors or prosthetic groups of different enzymes, with essential roles in both cellular and molecular functions. The most active vitamins are biotin, folate, nicotinamide, pantothenic acid, riboflavin, thiamine and vitamin B12. Although the necessity of very low concentrations of this component, the presence of VITs in-vitro is key and their absence may lead to decrease cell growth, death or loss of function. Therefore, we add vitamins to our BACTs in order to improve local cell homeostasis and survival. ${ }^{21}$

\subsection{Reduced Glutathione (GSH)}

GSH is one of the most powerful antioxidants in nature and can support cell survival antagonizing the negative effect of Reactive Oxygen Species (ROS) on grafted ADSCs. Other means to oppose the oxidative stress on cell grafts, such as deferoxamine and $\mathrm{N}$-acetylcysteine, has been also proposed, ${ }^{22,23}$ but GSH is the most important and versatile 
endogenous ROS scavenger. ${ }^{24}$ Used in the past in specific clinical situations, such as acute poisoning and severe postoperatory stress, both per os and IV administration, today is often used off label as ancillary therapy in the most different cases from cataract and glaucoma to skin whitening. The authors proposed a novel use by directly adding GSH to the BACTs mixture (200 $\mathrm{mg}$ in $2 \mathrm{ml}$. diluent) in order to counterbalance the oxidative stress occurring in the recipient site after the grafting.

\subsection{TYPE 2: Morphogenic proteins}

Bioactive Morpho-Proteins can be added to BACTs to stimulate cells' recruitment and redirecting towards specific cellular lines and to support their intake to the host recipient site. For the clinical treatment, for example, of osteoarthritis Type 2 the addition of 1500 I.U. of Bone Morpho Protein 2 (BMP-2) to BACTs drives ADSCs precursors toward the chondrogenic lineage, boosting the cartilage regeneration for the clinical treatment. ${ }^{25}$ This possibility could be very interesting, especially for those cases in which the regenerative action should be focused toward a specific tissutal target.

\subsection{Carriers}

Carriers have the ability to improve cellular structure and graft's implant. The HA (Hyaluronic Acid), the PA (Polycaproic Acid) and the ECM (Extra Cellular Matrix) represent important carriers within this field. The HA has a fundamental role in tissue volumization and regeneration. At the beginning of our in-vitro works, we mainly used a buffer-type HA $(\mathrm{pH}>7,1)$ because the traditional HA with a $\mathrm{pH}$ between 6.6 and 6.7 was negatively interfering with niches' preservation and function. Even the PA $\mathrm{CH}_{3}\left(\mathrm{CH}_{2}\right)_{4} \mathrm{COOH}$, an hexanoic acid derived from the hexane $(\mathrm{pH}=7)$, was proven to be effective. However, we never obtained satisfactory results both with HA or with PA.

At present, the recipient ECM is the main carrier of our BACTs. Its three-dimensional network is composed by collagen, enzymes and glycoproteins. Due to its features in cell adhesion, cell-to-cell communication and differentiation, the ECM currently represents the most reliable and user-friendly tool to facilitate the reimplantation of the graft while supporting the structure of the other bioactive components.

\subsection{Clinical application}

Translating the in-vitro technical steps into the clinical daily setting for the scope of Regenerative action authors, in close collaboration with many other specialists, have structured different novel protocols, using different bio-components concentration and proportions, to be applied in many clinical fields and specialties. Plastic Surgery for Breast Reconstruction, Microsurgery, Wound Healing, Diabetic foot and Burns. Orthopedic Surgery (OS), Rheumatology (RM), Uro-Gynecology (UG), Dermatology (DM) and Pain Management (PM) are some of the many other surgical specialties getting great benefits from concrete clinical applications of BACTs strategies. The regenerative potential of these new strategies can definitely be of great help in many other critical clinical situation. Among others new application the endobronchial administration of freshly isolated ADSCs was demonstrated safe ${ }^{26}$ and during this ongoing COVID 19 pandemia, we started using dedicated REMIX bio active Mixtures for stimulating and supporting the repair of pulmonary interstitial fibrosis. The paracrine modulatory action of MSCs on lung cells together with their ability to differentiate favoring the repair of tissue lesions are the mechanisms of action limiting scarring and promoting regeneration.

\subsection{BACTs in Orthopedic Surgery}

Orthopedic Surgery is definitely the surgical specialty that most can benefit of these new therapeutic strategies. At the present time the use of basic and advanced regenerative procedures in Orthopedic Surgery is estimated close to $30 \%$ of the total number of procedures globally performed (3 times more than in Plastic Surgery). The possibility to improve joints mobility and pain control meanwhile stimulating cartilage regeneration in OA type 1 and 2 , or to speed up recovery and healing processes after acute or chronic trauma and tendons reconstruction or to improve bone consolidation in complex fractures is concrete. The different protocols are named REMIX (acronym for Regenerative Mixture) and are identified by specialty and type of the composition (i.e, REMIX PS Type $1 / 2 / 3$ or REMIX OS Type 1 / 2); the cellular components (SVF and PRF) are expressed in percentages while the other additional components (AA, Vitamins, GSH) are expressed in mgs $x$ cc. The REMIX OS Type 1 for joints regeneration in OA type 1 and 2, is composed of $80 \%$ of freshly isolated SVF, 20\% PRF, , $25 \mathrm{mgs} x$ cc of Aminoacids, $150 \mathrm{mgs}$ $\mathrm{x}$ cc. of Vitamins and $20 \mathrm{mg} \mathrm{x}$ cc of reduced Glutathion (GSH). Whenever necessary to stimulate a chondrogenic cells recruitment it is possible to add to the regenerative mixture 1500 I.U. of Bone Morphoprotein 2 (BMP2). ${ }^{26}$ These components are mixed together and grafted, strictly under echo guidance, to the recipient site.Table 3

We recently carried on a comparative study from January 2019 to march 2020 on 22 patients affected by bilateral knee joints Osteoarthritis type 1 and 2. Patients were treated on one side with freshly isolated adipose derived SVF only, while the contralateral side was treated with BACTs REMIX Ortho Type 1.

Pain Control and Joint Function has been assessed at 30, 90 and 180 days after local treatment by using the VAS Pain Score and WOMAC Osteoarthritis Index. A significant improvement in both pain control and joint function in the 
Table 3: The components are mixed together and grafted under echo guidance into the recipient site which is acting as a real "in vivo bio-reactor".

\begin{tabular}{ll}
\hline $\begin{array}{l}\text { REMIX Ortho Type } 1 \text { for Knee's Joints Regeneration (15 } \\
\text { cc.) }\end{array}$ \\
CSVF & $80 \%$ \\
PRF & $20 \%$ \\
BMP2 & $1500 \mathrm{I} . \mathrm{U}$. \\
Aminoacids & $25 \mathrm{mgs} \mathrm{x} \mathrm{cc}$ \\
Vitamins & $150 \mathrm{mgs} \times \mathrm{cc}$ \\
GSH & $20 \mathrm{mg} \mathrm{x} \mathrm{cc}$ \\
\hline
\end{tabular}

side treated with BACTs has been clearly demonstrated. Table 4.

Table 4: Comparative Study on 22 patients suffering for bilateralknees joint AO type 1/2: Patients were treated in one side with fresh adipose derived SVF and in the contralateral side with the REMIX Ortho Type 1.

\begin{tabular}{lll}
\hline SVF vs. REMIX Knee's Ostheo Arthritis type 1 \\
& SVF & REMIX \\
30 days & 31\% Pain control & 53\% Pain control \\
& improvement & improvement \\
& 23\% Function & 39\% Function \\
& improvement & improvement \\
$\mathbf{9 0}$ days & 36\% Pain ontrol & 62\% Pain control \\
& improvement & improvement \\
& 29\% Function & 44\% Function \\
& improvement & improvement \\
$\mathbf{1 8 0}$ days & 36\% Pain control & 73\% Pain control \\
& improvement & improvement \\
& 30\% Function & 53\% Function \\
& improvement & improvement \\
\hline
\end{tabular}

- Pain control improvement assessed by VAS Scott-Hutchinson Scale;

\subsection{Ongoing researches and new perspectives}

As mentioned before, there are unfortunately many factors, which can heavily interfere with Regeneration and cell integration into the recipient site. In addition to the abovedescribed strategies to improve Transplanted Cells survival, the authors are actively working on new lines of research in order to improve the regenerative response. The redirection of cytokines pools into a probiotic pathway, the stimulation of MUSE cells population through a specific donor site preparation and delayed harvesting phase, ${ }^{27}$ Photo-Bio Modulation, extraction and concentration of autologous and allogenic Exosomes ${ }^{28}$ and exertional gene expression are important pillars of our new strategies destinated to further expand the clinical applications and outcomes [Table 5].

\section{Conclusions}

The most important limit of current regenerative approaches is that scientists and medical professionals have the unavoidable obligation to comply with rules, laws and
Table 5: FuturePerspectives and new strategies

\begin{tabular}{ll}
\hline Strategies & Effect \\
Jak Kinases inhibitors & Cytokines Modulation \\
Donor Site Preparation & Cells/ECM Enrichment \\
Delayed Harvesting & MUSE Stimulation \\
MUSE Cells & Cellular Boost \\
Photo-biomodulation & Cellularity and Replication \\
Exosomes & Paracrine Factors
\end{tabular}

regulations becoming every day more severe and restrictive. Hence, these procedures cannot exploit the possible benefits of other powerful tools such as tissue engineering techniques and of pro-survival gene transfer procedures.

Nonetheless, surgeons and scientists involved in regenerative medicine should focus on affordable strategies allowing their diffuse application in all the settings of treatment, avoiding the utilization of very expensive technologies with none or minimal improvement of cells isolation and concentration outcome.

Moreover, the concrete possibility to boost the stemness of regenerative grafts is still limited by the fact that HGM procedures, such as mesenchymal stem cell cultivation and expansion, are still not legally allowed in most of the Countries. For all these reasons, we do not have the possibility to deeply analyze the in-vivo proliferation and differentiation levels of our regenerative mixtures.

Even if cells sorting and immunophenotyping evaluations cannot be performed for each single patient, dependable and reliable technologies should be offered to our patients. Minimal values of the most important parameters of regenerative strategies, such as nucleated cells concentration, viability and percentage of mesenchymal progenitors, type and concentration of GFs should be better defined by the international scientific community.

The fresh regenerative mixture induces the in-vivo recipient site to act as a real bioreactor. Future preclinical and clinical studies are needed in order to define the best recruiting activity, cell sorting, differentiation and cellularity to the recipient sites. In addition, further study will aid in defining the best REMIX (REgenerative MIXture) for different clinical needs and anatomical districts.

In addition to the above-described strategies to improve Transplanted Cells survival, the author is now working on new lines of researches in order to improve the regenerative response such as the redirection of cytokines pools into a probiotic pathway and the stimulation of MUSE cells through a specific donor site preparation and a delayed harvesting phase. Even Photo-Bio Modulation and exertional gene expression are important pillars of these new strategies. Sharing this new approach will definitely improve the efficacy of regenerative medicine procedures respecting regulatory frames related to cell manipulation limits for the safety of our patients. 
The data merging from our experience in the last 4 years are extremely promising but it's now mandatory to further confirm the solid rationale behind this new exciting line of research providing additional data and further elucidate the therapeutic value of using this new Regenerative strategy to consolidate outcomes and results. It is easy to predict that shared guidelines should be established, in close collaboration with many other specialists such as Orthopedic Surgeons, for standardizing typology and proportion of the different regenerative components to be adapted to the different clinical and anatomical situations, depending on whether the need to stimulate the regenerative processes or to modulate the inflammatory response is prevalent.

\section{Conflicts of Interest}

The authors declare that there are no conflicts of interest regarding the publication of this paper.

\section{Source of Funding}

None.

\section{References}

1. Mason C, Brindley DA, Culme-Seymour EJ, Davie NL. Cell therapy industry: billion dollar global business with unlimited potential. Regen Med. 2011;6(3):265-72. dor:1022

2. I CAI. Review: mesenchymal stem cells: cell-based reconstructive therapy in orthopedics. Tissue Eng. 2005;11(7-8):1198-211.

3. Galipeau J, Sensébé L. Mesenchymal Stromal Cells: Clinical Challenges and Therapeutic Opportunities. Cell Stem Cell. 2018;22(6):824-33.

4. Trohatou O, Roubelakis MG. Roubelakis, Mesenchymal Stem/Stromal Cells in Regenerative Medicine: Past, Present, and Future. Cell Reprogram. 2017;19(4):217-24.

5. Trohatou O, Anagnou NP, Roubelakis MG. Human amniotic fluid stem cells as an attractive tool for clinical applications. Curr Stem Cell Res Ther. 2013;8(2):125-32.

6. Crisan M, Yap S, Casteilla L, Chen CW, Corselli M, Park TS, et al. A perivascular origin for mesenchymal stem cells in multiple human organs. Cell Stem Cell. 2008;3(3):301-13.

7. Rubin JP, Defail A, Rajendran N, Marra KG. Encapsulation of adipogenic factors to promote differentiation of adipose-derived stem cells. J Drug Target. 2009;17(3):207-215.

8. Yoshimura K, Shigeura T, Matsumoto D, Sato T, Takaki Y, AibaKojima E, et al. Characterization of freshly isolated and cultured cells derived from the fatty and fluid portions of liposuction aspirates. $J$ Cell Physiol. 2006;208(1):64-76. 101:10.1002/jcp.20636.

9. Sthijns M, Van Blitterswijk CA, Lapointe VLS. Redox regulation in regenerative medicine and tissue engineering: The paradox of oxygen. J Tissue Eng Regen Med. 2018;12(10):2013-20.

10. Lu Z, Chen Y, Dunstan C, Roohani-Esfahani S, Zreiqat $\mathrm{H}$ Priming Adipose Stem Cells with Tumor Necrosis Factor-Alpha Preconditioning Potentiates Their Exosome Efficacy for Bone Regeneration. Tissue Eng Part A. 2017;23(21-22):1212-20.

11. Zocchi ML, Vindigni V, Pagani A, Pirro O, Conti G, Sbarbati A, et al. Regulatory, ethical, and technical considerations on regenerative technologies and adipose-derived mesenchymal stem cells. Eur $J$

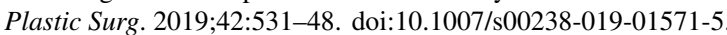

12. Zocchi ML. Regenerative assisted microsurgery (RAM) and regenerative assisted supermicrosurgery (RASM): the future of microsurgery? Eur J Plast Surg. 2021;p. 1-3. 10ن-10.1007/s00238 (12-018ा2-6
13. Bora P, Majumdar AS. Adipose tissue-derived stromal vascular fraction in regenerative medicine: a brief review on biology and translation. Stem Cell Res Ther. 2017;8(1):145. 101:10.186/s 132877 017-0598-y

14. Si Z, Wang X, Sun C, Kang Y, Xu J, Wang X, et al Adipose-derived stem cells: Sources, potency, and implications for regenerative therapies. Biomed Pharmacother. 2019;114:108765. do1:10.1016/1.b10pha.2019.108765

15. Kobayashi E, Flückiger L, Fujioka-Kobayashi M, Sawada K, Sculean A, Schaller B, et al. Comparative release of growth factors from PRP, PRF, and advanced-PRF. Clin Oral Investig. 2016;20(9):2353-60.

16. Steller D, Herbst N, Pries R, Juhl D, Hakim SG. Impact of incubation method on the release of growth factors in non-Ca(2+)-activated PRP, $\mathrm{Ca}(2+)$-activated PRP, PRF and A-PRF. J Craniomaxillofac Surg. 2019;47(2):365-72.

17. Caruana A, Savina D, Macedo JP, Soares SC. From Platelet-Rich Plasma to Advanced Platelet-Rich Fibrin: Biological Achievements and Clinical Advances in Modern Surgery. Eur $J$ Dent. 2019;13(2):280-6.

18. Vahabi S, Vaziri S, Torshabi M, Esfahrood ZR. Effects of Plasma Rich in Growth Factors and Platelet-Rich Fibrin on Proliferation and Viability of Human Gingival Fibroblasts. J Dent. 2015;12(7):504-12.

19. Choukroun J, Diss A, Simonpieri A, Girard MO, Schoeffler C, Dohan SL, et al. Platelet-rich fibrin (PRF): a second-generation platelet concentrate. Part IV: clinical effects on tissue healing. Oral Surg Oral Med Oral Pathol Oral Radiol Endod. 2006;101(3):e56-60.

20. Salazar A, Keusgen M, Hagen J. Amino acids in the cultivation of mammalian cells. Amino Acids. 2016;18(5):1161-71.

21. Godoy-Parejo C, Deng C, Zhang Y, Liu W, Chen G. Roles of vitamins in stem cells. Cell Mol Life Sci. 2020;77(9):1771-91.

22. Pietruski P, Paskal W, Paluch Ł, Paskal AM, Nitek Z, Włodarski P. The Impact of N-Acetylcysteine on Autologous Fat Graft: First-in-Human Pilot Study. Aesthetic Plast Surg. 2020;.

23. Temiz G, Sirinoglu H, Yesiloglu N, Filinte D, Kaçmaz C. Effects of Deferoxamine on Fat Graft Survival. Facial Plast Surg.

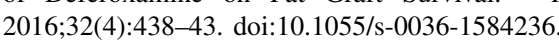

24. Hatem E, Berthonaud V, Dardalhon M, Lagniel G, Baudouin-Cornu P, Huang ME, et al. Free Radic Biol Med. 2014;75(1):S25-6 doi:10.1016/j.freeradbiomed.2014.10.746.

25. Khan SN, Lane JM. The use of recombinant human bone morphogenetic protein-2 (rhBMP-2) in orthopaedic applications. $\quad$ Expert Opin Biol Ther. 2004;4(5):741-8.

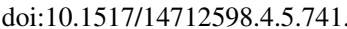

26. Tzouvelekis A, Paspaliaris V, Koliakos G, Ntolios P, Bouros E, Oikonomou A, et al. A prospective, non-randomized, no placebocontrolled, phase $\mathrm{Ib}$ clinical trial to study the safety of the adipose derived stromal cells-stromal vascular fraction in idiopathic pulmonary fibrosis. J Transl Med. 2013;11:171. 10i:10 186/479Б876-11-17]

27. Tatsumi K, Kushida Y, Wakao S, Kuroda Y, Dezawa M. Protocols for Isolation and Evaluation of Muse Cells. Adv Exp Med Biol. 2018;1103:69-101. d01:10.1007/9/8-4-431-56847-6 4.

28. Nakamura Y, Miyaki S, Ishitobi H, Matsuyama S, Nakasa T, Kamei $\mathrm{N}$, et al. Mesenchymal-stem-cell-derived exosomes accelerate skeletal muscle regeneration. FEBS Lett. 2015;589(11):1257-65.

\section{Author biography}

Michele Zocchi, Adjoint Professor,

Institute of Plastic, Reconstructive and Regenerative Surgery. University of Padua - Italy

Cite this article: Zocchi M. New strategies in regenerative medicine and surgery and their application to orthopedic surgery field: The bio-active composite therapies (BACTs). IP Int J Orthop Rheumatol 2021;7(2):58-64 